\title{
Classical Resummation and Breakdown of Strong-Field QED
}

\author{
T. Heinzlø, ${ }^{*}$ A. Ilderton, ${ }^{\dagger}$ and B. King $\oplus^{\ddagger}$ \\ Centre for Mathematical Sciences, University of Plymouth, Plymouth PL4 8AA, United Kingdom
}

(Received 5 February 2021; revised 11 May 2021; accepted 30 June 2021; published 5 August 2021)

\begin{abstract}
QED perturbation theory has been conjectured to break down in sufficiently strong backgrounds, obstructing the analysis of strong-field physics. We show that the breakdown occurs even in classical electrodynamics, at lower field strengths than previously considered, and that it may be cured by resummation. As a consequence, an analogous resummation is required in QED. A detailed investigation shows, for a range of observables, that unitarity removes diagrams previously believed to be responsible for the breakdown of QED perturbation theory.
\end{abstract}

DOI: 10.1103/PhysRevLett.127.061601

Examining the transition to classical physics can help us understand quantum theories, with topical examples being the classical post-Minkowskian expansion of general relativistic dynamics [1,2], classical double copy [3,4], and decoherence [5]. Whether classical or quantum, theories containing strong background fields are typically analyzed in the Furry expansion [6] (background field perturbation theory $[7,8]$ ) where the background is treated exactly, while particle scattering on the background is treated perturbatively. For quantum electrodynamics (QED) in strong fields, this amounts to employing the usual perturbative loop expansion in the fine structure constant $\alpha \ll 1$, while fermion propagators are "dressed" by the background. The Furry expansion is an essential tool in theory, experimental modeling, and numerical schemes used in astrophysics and plasma physics $[9,10]$.

However, the Ritus-Narozhny (RN) conjecture suggests that the Furry expansion breaks down for sufficiently strong fields $[11,12]$, because the perturbative coupling becomes enhanced by the field strength. In constant fields, for which the conjecture was originally formulated, one finds that the effective expansion parameter is not $\alpha$, but $\alpha \chi^{2 / 3}$ [13], where $\chi=\xi \eta$, the product of background field strength and particle energy invariants, $\xi$ and $\eta$, to be defined below (the latter being linearly related to the Mandelstam invariant $s$, see [14] and Supplemental Material, Sec. A [15]). The conjecture has been interpreted to hold for any background that is approximately "locally constant," i.e., constant over typical "formation scales" $[13,23]$. Because of the widespread use of the Furry expansion it is crucial to understand its regime of applicability [24-28].

Published by the American Physical Society under the terms of the Creative Commons Attribution 4.0 International license. Further distribution of this work must maintain attribution to the author(s) and the published article's title, journal citation, and DOI. Funded by SCOAP ${ }^{3}$.
The regime associated with the conjecture includes that of high-field strength and low energy [29,30], in which we can approximate QED by its classical limit. In invariant terms, low energy means $\eta \ll 1$. Here we will show explicitly how and where the breakdown of perturbation theory occurs in the classical limit, and that it does so in the more realistic case of nonconstant fields. More progress is possible in the classical theory, as we can effectively resum the classical perturbative series to all orders. We will show that this resummation cures the unphysical behavior associated with the breakdown of perturbation theory. In this way we can see how the RN conjecture is resolved, in the considered regime.

We emphasize that every term in the classical limit corresponds to some (collection of) terms in QED, and that the connection between them is nontrivial. Contrary to common lore, photon loops contribute in a subtle way to the classical limit [31-33]. Thus by investigating the classical limit we can learn about QED. Indeed, our results have direct implication for the quantum theory: we find that perturbation theory breaks down at far lower intensities than predicted by the RN conjecture.

Classical.-A strong background, $f^{\mu \nu}=e F_{\mathrm{ext}}^{\mu \nu} / m$, for $m$ and $e$ the electron mass and charge respectively, is characterized by a dimensionless coupling $\xi \sim f / \omega \gg 1$, for $\omega$ a typical frequency scale of the background. The classical equations of motion in such a background are

$$
\ddot{x}^{\mu}=\left(f^{\mu \nu}+e F^{\mu \nu} / m\right) \dot{x}_{\nu}, \quad \partial_{\mu} F^{\mu \nu}=j^{\nu},
$$

in which $F$ is the generated radiation field, $x^{\mu}$ is the particle orbit, and $j^{\nu}$ its current. (Note that $c=1$ throughout.) The classical limit of the Furry expansion corresponds to treating $f$, therefore $\xi$, exactly, and $e$ (made appropriately dimensionless) perturbatively. The zeroth order equations describe the Lorentz orbit in the background $f$, with no radiation. At higher orders, radiation and radiation backreaction (RR) appear [23,34-39]. The assumption behind 
the Furry expansion is simply that these RR corrections, corresponding to higher powers of $\alpha$ in QED, are subleading. It is known, though, that this is not always the case, and RR effects treated perturbatively can become large. We now give two examples showing that this behavior is unphysical, signaling the classical breakdown of the perturbative expansion.

First, an electron in a rotating electric field $\mathbf{E}(t)=$ $E_{0}(0, \cos \omega t, \sin \omega t)$ can, as shown in [40], have a closed orbit, with energy $m \gamma$ determined by $\xi=|e| E_{0} /(m \omega)$ and $\omega$. The Lorentz force prediction for the energy is $\gamma^{2}-1=\xi^{2}$. Furry picture perturbative corrections to this are not given by an expansion in a small parameter, though, but rather in powers of $\epsilon_{\mathrm{rad}} \xi^{3}$, where $\epsilon_{\mathrm{rad}}:=(2 / 3)\left(e^{2} / 4 \pi\right)(\omega / m)$, with leading behavior

$$
\gamma^{2}-1 \sim \xi^{2}\left(1-\epsilon_{\mathrm{rad}}^{2} \xi^{6}+\cdots\right) .
$$

Hence, for sufficiently strong fields, the corrections become larger than the supposedly dominant Lorentz force contribution and the perturbative expansion breaks down, signaled in (2) by the unphysical result $\gamma^{2}-1<0$.

For our second example, consider an electron in an arbitrary plane wave (direction $n_{\mu}$, typical frequency $\omega$, $k_{\mu}:=\omega n_{\mu}$, phase $\phi=k \cdot x$ ) with transverse electric field $\mathrm{a}^{\prime}(\phi)$. According to the Lorentz force, i.e., zeroth order in perturbation theory, the initial lightfront energy component $n \cdot p$ of the electron is conserved. The first perturbative correction to the final electron momentum $p_{\text {out }}$ is $[41,42]$

$$
\frac{n \cdot p_{\text {out }}}{n \cdot p}=1-\frac{2}{3} \frac{e^{2}}{4 \pi} \frac{k \cdot p}{m^{4}} \int d \phi\left|\mathbf{a}^{\prime}(\phi)\right|^{2} \equiv 1-\Delta .
$$

The effective expansion parameter is $\Delta \propto \xi^{2}$, which again may not be small; the expansion breaks down for $\xi \gg 1$, signaled here by the unphysical behavior $n \cdot p_{\text {out }}<0$.

In some cases it is possible to explicitly resum perturbative solutions to (1) [43]. A more general approach is to effectively resum the perturbative series by eliminating the electromagnetic variables from (1) to obtain the exact Lorentz-Abraham-Dirac (LAD) equation for the electron orbit [44]. For our first example, LAD implies that $\gamma$ satisfies the equation $\xi^{2}=\left(\gamma^{2}-1\right)\left(1+\epsilon_{\mathrm{rad}}^{2} \gamma^{6}\right)$ [40]. This recovers (2) if $\epsilon_{\text {rad }}$ is treated perturbatively, but behaves as $\gamma^{2}-1 \sim \xi^{2}\left(\epsilon_{\mathrm{rad}} \xi^{3}\right)^{-1 / 2}$ for $\epsilon_{\mathrm{rad}} \xi^{3} \gg 1$, i.e., resummation corrects the unphysical behavior of perturbation theory. For plane waves, the solution to the LAD equation is not known, so we use the Landau-Lifshitz (LL) equation instead [45], which agrees exactly with LAD to low orders and is adequate classically [46,47]. (What is important is that both LAD and LL equations provide all-order results.) The exact solution to LL yields [41]

$$
\frac{n \cdot p_{\text {out }}}{n \cdot p}=\frac{1}{1+\Delta}>0
$$

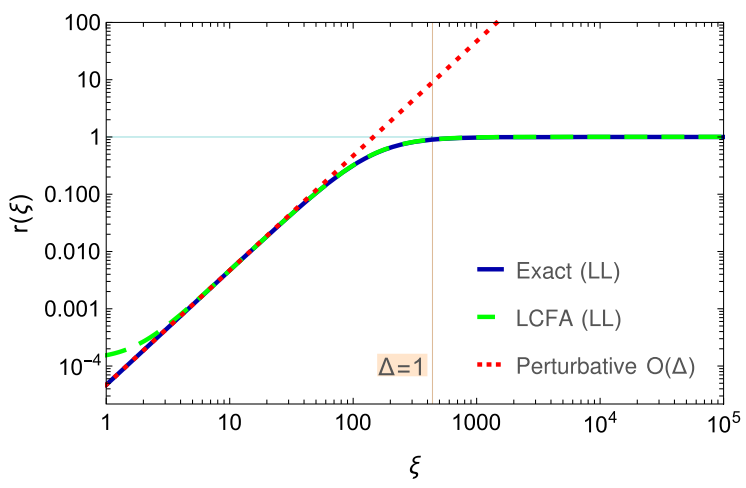

FIG. 1. Proportion $r(\xi)$, of initial electron energy radiated in a four-cycle circularly polarized plane wave pulse $\mathrm{a}(\phi)=m \xi \sin ^{2}(\phi / 8)\{\cos \phi, \sin \phi\}$ for $\phi \in[0,8 \pi]$ and $\mathrm{a}(\phi)=0$ otherwise. The radiated energy is bounded above in the LL result, but is unbounded for the perturbative, $O(\Delta)$, result. The LCFA discussed in the text is also shown: it characteristically overpredicts the radiated energy for $\xi \sim \mathcal{O}(1)$.

so that, comparing to (3) [42], resummation again fixes the unphysical behavior of perturbation theory.

Note that the coefficient of the integral in (3) is $(2 / 3) \alpha \eta$, with the QED energy invariant $\eta:=\hbar k \cdot p / m^{2}$. This underlines that both perturbative and resummed results have their origin in QED [31]. To begin making connections to QED we first need to understand in more depth what changes when we go from low orders of perturbation theory to allorders results. To do so we consider the energy-momentum $K_{\mu}$ radiated by an electron in a plane wave. This is calculated by inserting the LL solution for the electron orbit into the fully relativistic Larmor formula [47]. We focus for simplicity on the lightfront momentum fraction $r:=n \cdot K / n \cdot p$.

In Fig. 1, we show how the ratio $r$ depends on the intensity of a pulsed plane wave. The leading, $\mathcal{O}(\Delta)$, result scales without bound as $r \sim \Delta \sim \xi^{2}$; to this order in $\Delta$ one has that $r=1-n \cdot p_{\text {out }} / n \cdot p$, hence reaching $r>1$ reflects the unphysical behavior in (3). The behavior of the all-orders, or resummed, result, is completely different: the total energy radiated is bounded by $r \leq 1$, as demonstrated by the plateau in Fig. 1. Thus the effect of resummation is very clear, and physically sensible, but to help understand it we analyze the formation of the emitted radiation as a function of phase $\phi$. Following the established procedure of expanding double phase integrals in the difference of two phases [48,49], we develop a locally constant field approximation (LCFA) for our LLcorrected observables. Let $\Delta(\phi)$ be defined as in (3) but with the integral extending only up to $\phi$, and define $\mathcal{R}:=1+\Delta(\phi)$. Then we find (see Supplemental Material, Sec. B for details [15]) the classically resummed LCFA result

$$
\frac{d r}{d \phi}=-\frac{e^{2}}{4 \pi} \frac{m^{2}}{k \cdot p} \int_{0}^{\infty} d \bar{s} \bar{s} \mathcal{R}^{2}\left[\operatorname{Ai}_{1}(z)+\frac{2}{z} \operatorname{Ai}^{\prime}(z)\right],
$$


where $z=\left(\bar{s} \mathcal{R}^{2} / \bar{\chi}\right)^{2 / 3}, \bar{\chi}=\left|\mathbf{a}^{\prime}\right| k \cdot p / m^{3}$, and $\bar{s}=n \cdot k_{\text {out }} / n$. $p$ for $k_{\text {out }}$ the radiation wave vector. Note the simple relation $\bar{\chi}=\chi / \hbar$ relating the classical $\bar{\chi}$ to the quantum parameter $\chi$. The LCFA is benchmarked against the exact result and found to agree excellently in the high-field limit in Fig. 1.

If $\Delta \rightarrow 0$ ( $\mathcal{R} \rightarrow 1$ ), (5) tends to the classical limit of the $\mathcal{O}(\alpha)$ quantum result [23]. Let us recall that, in the corresponding quantum emission probability, the large- $\chi$ limit leads to small Airy function arguments. The crucial step is to assume that, in this limit, one can replace $\operatorname{Ai}^{\prime}\left(\{s /[\chi(1-s)]\}^{2 / 3}\right) \approx \operatorname{Ai}^{\prime}(0)$, in which $s=\hbar \bar{s}$ is the photon lightfront momentum fraction, $0 \leq s \leq 1$. This simplification results, ultimately, in the scaling of the probability with $\alpha \chi^{2 / 3}$. The assumption here is, note, that $s / \chi \equiv \bar{s} / \bar{\chi} \ll 1$, hence the RN scaling is actually associated with the smallness of a classical parameter $\bar{s} / \bar{\chi}$.

The RN scaling can be seen directly in the classical result for the lightfront momentum fraction, Eq. (5). The $\bar{s}$ integral in (5) can be written as an integral over a lowfrequency region, $\bar{s} \leq 1$, plus a high-frequency region, $\bar{s}>1$. The integral over the low-frequency region is exactly equal to the $\mathcal{O}(\alpha)$ quantum result with recoil and spin set to zero. Hence, just like the QED result, it scales as $\bar{\chi}^{2 / 3} \sim \xi^{2 / 3}$ in the high-field limit typical of the $\mathrm{RN}$ conjecture. However, the high-frequency $(\bar{s}>1)$ contribution grows with a larger power, $\sim \xi^{2}$, and thus dominates the scaling of the classical rate (5) in agreement with previous expectations $[34,50]$.

In Fig. 2 we plot the local rate (5) for various $\xi$, and compare to the perturbative (Lorentzian) result without RR. Figure 2(a) shows that the higher the pulse intensity, the earlier the majority of radiation is emitted and hence the quicker the electron is decelerated. Without RR, on the other hand, the rate of radiation is symmetric with the shape of the pulse: as much is emitted in the tail as in the rise. This is emphasized in Fig. 2(b), in which we pick two phase points early and late in the pulse $[\phi=2 \pi$ and $\phi=6 \pi$ as also indicated in Fig. 2(a)], and illustrate how the rate of emission at those points changes as $\xi$ is increased. The perturbative scaling, $\sim \xi^{2}$ at small $\xi$, is corrected at large $\xi$ to a scaling $\sim \xi^{-2}$ such that $r(\xi)$ never exceeds unity. Clearly, resummation in $\Delta$ has changed the large- $\xi$ behavior of the leading order, Lorentzian, result.

The origin of these different behaviors can be traced back to the impact of RR corrections on the Airy argument $z$ in (5): note that it is the behavior of the analogous argument in QED results which determines the large- $\xi$ asymptotic behavior. Here we have,

$$
\text { if } \Delta \ll 1: z \sim\left(\frac{\bar{s}}{\xi}\right)^{2 / 3} ; \quad \text { if } \Delta \gg 1: z \sim\left(\bar{s} \xi^{3}\right)^{2 / 3} \text {. }
$$

If RR corrections are neglected, large $\xi$ yields small Airy arguments $\sim(\bar{s} / \xi)^{2 / 3} \equiv(s / \xi)^{2 / 3}$, which leads to the
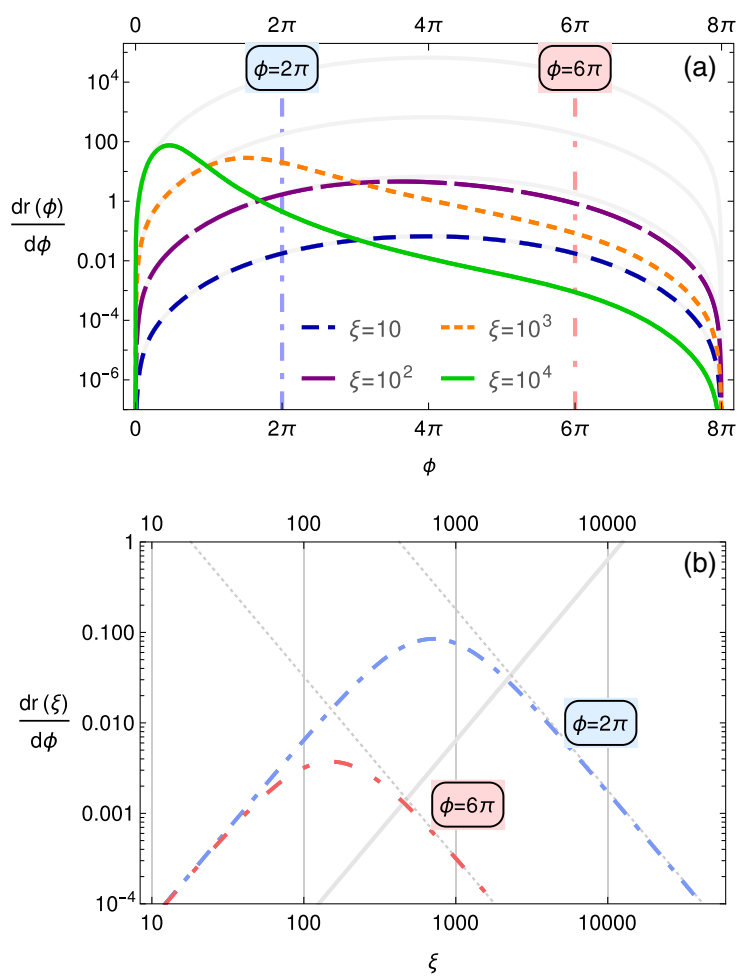

FIG. 2. (a) Classically resummed LCFA rate of radiation (5) (colored lines), compared to the $\mathcal{O}(\Delta)$ result (faint gray lines). (b) Intensity scaling of (5) at two fixed points in the pulse (on the rising and falling edge respectively). The same field and parameters were used as in Fig. 1.

power-law $\xi^{2}$ dependence of the total emitted radiation associated with the breakdown of perturbation theory ( $r>1$, recall Fig. 1). With RR, though, large $\xi$ yields a large Airy argument which suppresses $d r / d \phi$ (leading to $r \leq 1)$. Hence, crucially, resummation reverses the asymptotic limit of the Airy functions compared to that expected from perturbation theory. We saw the physical consequence of this reversal above: the rate of radiation in the high- $\xi$ region is suppressed as $\xi^{-2}$; hence even in high- $\xi$ pulses the radiation is mainly generated in the small- $\xi$ regions in the rising edge of the pulse, where the rate scales as $\xi^{2}$. The plateau in $r$ at ever higher intensities is a consequence of a balance between ever-stronger decelerations over evershorter durations.

The above examples advance our understanding of the RN conjecture significantly: we have seen that the breakdown of perturbation theory in strong fields appears even in nonconstant backgrounds, that it occurs classically, and that it can be resolved by classical resummation.

Quantum.-Quantum effects can become relevant, as intensity increases, before large classical RR effects set in [46]. As we saw below (5), this can change the power of $\xi$ or $\chi$ in perturbative results, reducing the energy radiated compared to classical predictions [50], but it does not prevent perturbative breakdown, so that resummation is still 
required. Comparing scales shows that resummation of classical effects becomes necessary when $\xi^{2} \sim 1 / \alpha \eta$, referred to as the radiation dominated regime [23]; what we have learned, though, is that this condition is really signaling the breakdown of the Furry expansion, and that it occurs at far lower intensities $\xi$ than required by the RN conjecture, $\xi^{2} \sim 1 / \alpha^{3} \eta^{2}$ (neglecting pulse length effects in both cases). This implies that the contributions which fix unphysical behavior in the QED Furry expansion must include at least those which fix its classical limit. In this light we reconsider some of the observables above, but now in QED.

The electron momentum after scattering is given in QED by the expectation value of the momentum operator, $\hat{P}_{\mu}$ in the final state $[51,52]$. In the Furry expansion in a plane wave background, the lowest order contribution to $\left\langle\hat{P}_{\mu}\right\rangle$ comes at $\mathcal{O}\left(\alpha^{0}\right)$ from elastic scattering, and yields $\left\langle\hat{P}_{\mu}\right\rangle=\pi_{\mu}$, the Lorentz force momentum of a classical electron which has traversed the wave [53]. This lowest order result receives corrections of order $\alpha$ or $e^{2}$ in the quantum and classical theory, respectively. The general structure of the quantum corrections is known to all orders in $\alpha[54,55]$ :

$$
\left\langle\hat{P}_{\mu}\right\rangle:=\sum_{f} d \mathbb{P}_{f}\left(\pi_{\mu}-\sigma_{\mu}(f)+\lambda(f) n_{\mu}\right),
$$

where $d \mathbb{P}_{f}$ is the all-loop differential probability to obtain some final state $f, \sigma_{\mu}(f)$ denotes the total momentum of particles produced during scattering, and momentum conservation fixes the scalar $\lambda(f)$ in terms of $\pi_{\mu}$ and $\sigma_{\mu}$ [55]. The nonperturbative result (6) can be simplified by noting that unitarity implies

$$
\sum_{f} d \mathbb{P}_{f}=\mathbb{P}\left(e^{-} \rightarrow \text { anything }\right)=1 .
$$

This leads to the statement (reminiscent of the nonrenormalization theorems of [56]) that the leading term, the Lorentz momentum, in (6) must have unit coefficient. Let us compare this with results in the RN literature, which has focused on self-energy corrections to elastic scattering (see $[57,58]$ for other processes). These contain, at $n$-loop order in a constant crossed field, terms that scale asymptotically as $\left(\alpha \chi^{2 / 3}\right)^{n}$ and contribute only to the first term in (6), hence to the unitarity sum (7), with coefficients $c_{n}$ as,

$$
\sum_{f} d \mathbb{P}_{f} \sim\left|1+\sum_{n=1} c_{n}\left(\alpha \chi^{2 / 3}\right)^{n}\right|^{2} \sim \chi^{2},
$$

with the final expression obtained through "bubble-chain" resummation [13]. The result (8) clearly violates unitarity (7). It is thus inconsistent to neglect other terms contributing to $\left\langle\hat{P}_{\mu}\right\rangle$ (even to elastic scattering), at each order in $\alpha$.
Moreover, these terms cancel, through unitarity, the offending powers of $\alpha \chi^{2 / 3}$ associated with self-energy loops (and any other deviation from unity).

This points to a previously unexplored mechanism by which parts of the Furry expansion are brought back under control. We can make this more explicit at lowest nontrivial order, that is $\mathcal{O}(\alpha)$, where there are two contributions to (6): a one-loop self-energy contribution and a tree-level onephoton emission contribution. It was already observed in [55] that there is a cancellation between these terms, which is essential for two reasons. First, it removes infrared divergences. Second, it is required for the classical limit to exist at all, as otherwise $\left\langle\hat{P}_{\mu}\right\rangle$ would contain, for general plane wave backgrounds, diverging terms of order $1 / \hbar$. What was not previously noted is that this same cancellation removes, for constant fields, or if using the LCFA, terms scaling as $\alpha \chi^{2 / 3}$. We have above been able to generalize that result to all orders.

The discussed cancellation holds also for variables without a classical analog, such as the variance in the momentum, defined by $\langle\hat{P}\rangle^{2}-\left\langle\hat{P}^{2}\right\rangle[37,59]$.

The above reinforces our findings for the classical theory: some of the QED terms previously identified as leading to perturbative breakdown actually drop out. Their RN scaling behavior thus becomes irrelevant, at least for the observables considered here. What remains in $\left\langle\hat{P}_{\mu}\right\rangle$ after such cancellations still needs to be resummed; it includes the classical limit as $\hbar \rightarrow 0$, including, e.g., (3) at $\mathcal{O}(\alpha)$ [54,55]. While it is still unknown how to perform this resummation generally, in QED, it has very recently been achieved in the classical limit [33].

Conclusions.-We have shown that the conjectured breakdown of perturbation theory in strong-field QED is not particular to the constant field case originally considered $[11,12]$. Furthermore, because the relevant physical domain is high-field strength, not high energy [29,30], the breakdown can be triggered classically, at lower intensities than suggested by the RN conjecture. Classical resummation then becomes necessary, and we have confirmed that this (achieved through the use of exact, or all-orders, results), can indeed fix the unphysical behavior of perturbation theory in the classical limit.

While our results suggest that the breakdown may be a generic feature of the Furry expansion, we note that some strong-field expansions can be convergent; an example is the Volkov solution, which has a convergent exponential series expansion for any field strength [60].

The implication of our results for strong-field QED is that in order to obtain physically sensible results at high $\chi$ one should resum at least all classical contributions at each loop order. The relevant diagrams include loops and photon emissions, cf. [57]. We have also seen, for several natural observables, that unitarity removes many previously considered diagrams scaling with powers of $\alpha \chi^{2 / 3}$. In the context of the RN conjecture, it may thus be inconsistent to 
look at only subsets of diagrams. Such issues have recently been highlighted through different subset resummations of Schwinger-Dyson equations [61].

We comment finally on the impact of resummation on photonic observables [62]. A probe photon in a strong background field can undergo helicity flip due to loop effects, scaling like $\alpha \chi^{2 / 3}$ at one loop, with higher loop corrections believed to scale with higher powers of the same [13]. The effect is purely quantum, but classical results are nevertheless relevant because higher loop corrections contain classical parts: cutting the loops gives the probability of pair production (a quantum effect) accompanied by photon emission from the created pair [63], which has a classical part. We now know that such classical effects need to be resummed in the high-field limit. Furthermore, the one-loop effect itself exponentiates to a phase, yielding vacuum birefringence of the probe [64], so again resummation gives physically sensible results.

The authors are supported by the EPSRC, Grant No. EP/ S010319/1 (A. I., B. K.), and the Leverhulme Trust, Grant No. RPG-2019-148 (T. H., A. I.).

*thomas.heinzl@plymouth.ac.uk †anton.ilderton@plymouth.ac.uk Address from 1st September: Higgs Centre for Theoretical Physics, University of Edinburgh, EH9 3FD, Scotland, United Kingdom.

*b.king@plymouth.ac.uk

[1] C. Cheung, I. Z. Rothstein, and M. P. Solon, Phys. Rev. Lett. 121, 251101 (2018).

[2] Z. Bern, C. Cheung, R. Roiban, C.-H. Shen, M. P. Solon, and M. Zeng, Phys. Rev. Lett. 122, 201603 (2019).

[3] R. Monteiro, D. O'Connell, and C. D. White, J. High Energy Phys. 12 (2014) 056.

[4] L. de la Cruz, B. Maybee, D. O'Connell, and A. Ross, J. High Energy Phys. 12 (2020) 076.

[5] M. Schlosshauer, Phys. Rep. 831, 1 (2019).

[6] W. H. Furry, Phys. Rev. 81, 115 (1951).

[7] B. S. DeWitt, Phys. Rev. 162, 1195 (1967).

[8] G. 't Hooft, in Functional and Probabilistic Methods in Quantum Field Theory. 1. Proceedings, 12th Winter School of Theoretical Physics, Karpacz (1975), pp. 345-369.

[9] R. Turolla, S. Zane, and A. L. Watts, Rep. Prog. Phys. 78, 116901 (2015).

[10] A. Gonoskov, S. Bastrakov, E. Efimenko, A. Ilderton, M. Marklund, I. Meyerov, A. Muraviev, A. Sergeev, I. Surmin, and E. Wallin, Phys. Rev. E 92, 023305 (2015).

[11] V. Ritus, Sov. Phys. JETP 30, 1181 (1970), http://www.jetp .ac.ru/cgi-bin/e/index/e/30/6/p1181?a=list.

[12] N. B. Narozhnyi, Phys. Rev. D 21, 1176 (1980).

[13] A. A. Mironov, S. Meuren, and A. M. Fedotov, Phys. Rev. D 102, 053005 (2020).

[14] T. Heinzl and A. Ilderton, Opt. Commun. 282, 1879 (2009).

[15] See Supplemental Material at http://link.aps.org/ supplemental/10.1103/PhysRevLett.127.061601 for a discussion of the parameter regime relevant to the RN conjecture, the derivation of the classical LCFA, and an analysis of one loop contributions in the classical limit, which includes Refs. [16-22].

[16] D. Seipt and B. King, Phys. Rev. A 102, 052805 (2020).

[17] T. W. B. Kibble, A. Salam, and J. A. Strathdee, Nucl. Phys. B96, 255 (1975).

[18] A. Di Piazza, M. Tamburini, S. Meuren, and C. H. Keitel, Phys. Rev. A 98, 012134 (2018).

[19] L. Bieri and D. Garfinkle, Classical Quantum Gravity 30, 195009 (2013).

[20] D. Seipt, in Quantum Field Theory at the Limits: From Strong Fields to Heavy Quarks (2017), https://bib-pubdb1 .desy.de/record/330321.

[21] A. Ilderton and G. Torgrimsson, Phys. Rev. D 87, 085040 (2013).

[22] G. Torgrimsson, arXiv:2105.02220.

[23] A. Di Piazza, C. Muller, K. Z. Hatsagortsyan, and C. H. Keitel, Rev. Mod. Phys. 84, 1177 (2012).

[24] V. Yakimenko et al., Phys. Rev. Lett. 122, 190404 (2019).

[25] T. G. Blackburn, A. Ilderton, M. Marklund, and C.P. Ridgers, New J. Phys. 21, 053040 (2019).

[26] C. Baumann, E. N. Nerush, A. Pukhov, and I. Y. Kostyukov, Sci. Rep. 9, 9407 (2019).

[27] A. Di Piazza, T. N. Wistisen, M. Tamburini, and U. I. Uggerhøj, Phys. Rev. Lett. 124, 044801 (2020).

[28] L. Fedeli, A. Sainte-Marie, N. Zaïm, M. Thévenet, J.-L. Vay, A. Myers, F. Quéré, and H. Vincenti, arXiv:2012.07696.

[29] T. Podszus and A. Di Piazza, Phys. Rev. D 99, 076004 (2019).

[30] A. Ilderton, Phys. Rev. D 99, 085002 (2019).

[31] B. R. Holstein and J. F. Donoghue, Phys. Rev. Lett. 93, 201602 (2004).

[32] I. Huet, M. Rausch de Traubenberg, and C. Schubert, Proc. Sci. LL2018 (2018) 035.

[33] G. Torgrimsson, arXiv:2102.11346.

[34] A. Di Piazza, K. Z. Hatsagortsyan, and C. H. Keitel, Phys. Rev. Lett. 105, 220403 (2010).

[35] M. Vranic, J. L. Martins, J. Vieira, R. A. Fonseca, and L. O. Silva, Phys. Rev. Lett. 113, 134801 (2014).

[36] D. A. Burton and A. Noble, Contemp. Phys. 55, 110 (2014).

[37] V. Dinu, C. Harvey, A. Ilderton, M. Marklund, and G. Torgrimsson, Phys. Rev. Lett. 116, 044801 (2016).

[38] J. M. Cole et al., Phys. Rev. X 8, 011020 (2018).

[39] K. Poder et al., Phys. Rev. X 8, 031004 (2018).

[40] S. S. Bulanov, T. Z. Esirkepov, A. G. R. Thomas, J. K. Koga, and S. V. Bulanov, Phys. Rev. Lett. 105, 220407 (2010).

[41] A. Di Piazza, Lett. Math. Phys. 83, 305 (2008).

[42] C. Harvey, T. Heinzl, and M. Marklund, Phys. Rev. D 84, 116005 (2011).

[43] S. Zhang, Prog. Theor. Exp. Phys. 2013, 123A01 (2013).

[44] P. A. M. Dirac, Proc. R. Soc. A 167, 148 (1938).

[45] L. D. Landau, The Classical Theory of Fields, edited by E. M. Lifshitz, H. G. Schopf, and P. Ziesche (Elsevier, Amsterdam, 1987).

[46] S. V. Bulanov, T. Z. Esirkepov, M. Kando, J. K. Koga, and S. S. Bulanov, Phys. Rev. E 84, 056605 (2011).

[47] A. Di Piazza, Phys. Lett. B 782, 559 (2018).

[48] A. Ilderton, B. King, and D. Seipt, Phys. Rev. A 99, 042121 (2019). 
[49] A. Di Piazza, M. Tamburini, S. Meuren, and C. H. Keitel, Phys. Rev. A 99, 022125 (2019).

[50] T. Blackburn, Plasma Phys. 4, 5 (2020).

[51] V. Krivitsky and V. Tsytovich, Sov. Phys. Usp. 34, 250 (1991).

[52] A. Higuchi, Phys. Rev. D 66, 105004 (2002); 69, 129903(E) (2004).

[53] V. Dinu, T. Heinzl, and A. Ilderton, Phys. Rev. D 86, 085037 (2012).

[54] A. Ilderton and G. Torgrimsson, Phys. Rev. D 88, 025021 (2013).

[55] A. Ilderton and G. Torgrimsson, Phys. Lett. B 725, 481 (2013).

[56] C. Cheung and C.-H. Shen, Phys. Rev. Lett. 115, 071601 (2015).
[57] J. P. Edwards and A. Ilderton, Phys. Rev. D 103, 016004 (2021).

[58] A. Di Piazza and M. A. Lopez-Lopez, Phys. Rev. D 102, 076018 (2020).

[59] D. G. Green and C. N. Harvey, Phys. Rev. Lett. 112, 164801 (2014).

[60] T. W. B. Kibble, Phys. Rev. 138, B740 (1965).

[61] M. Borinsky, G. V. Dunne, and M. Meynig, arXiv: 2104.00593.

[62] F. Karbstein, Phys. Rev. Lett. 122, 211602 (2019).

[63] G. V. Dunne and Z. Harris, Phys. Rev. D 103, 065015 (2021).

[64] B. King and T. Heinzl, High Power Laser Sci. Eng. 4, E5 (2016). 